\title{
A77 A NOVEL TNFRSF1 GENE MUTATION IN A TURKISH FAMILY: REPORT OF THREE CASES
}

F Cosan, A Yazici, A Cefle, A Gul Division of Rheumatology, Kocaeli University, Turkey

10.1136/ard.2010.129619k

Background Tumour necrosis factor (TNF) receptor-associated periodic syndrome (TRAPS) is an autosomal dominantly inherited rare autoinflammatory disease. It caused by mutations in exon 2-3 and 4-5 of the TNFRSF1A gene on chromosome $12 \mathrm{p} 13.2$. TNFRSF1A gene encodes the $55 \mathrm{kDa}$ receptor for TNF. Attacks are associated with abdominal pain, myalgia, erythematous skin rash, conjunctivitis and periorbital oedema. More than 80 mutations have so far been identified. The authors report three patients of Turkish origin with TRAPS.

Patients and Methods The patients were followed in our outpatient clinic in Kocaeli University Division of Rheumatology. Because of their TRAPS-associated clinical features, the authors isolated genomic DNA from whole blood and sequenced the exon 2-3 and 4-5 third exon of TNFRSF1A gene after amplification with appropriate primers.

Results One of the patients with TRAPS, a 47-year-old woman, described recurrent attacks of fever, urticarial rash, conjunctivitis, athralgia, myalgia, abdominal pain, thoracic pain, headache, fatigue and elevated acute phase response since her childhood. With sequencing of the TNFRSF1A gene, the authors identified heterozygous C29R mutation which has not been reported before in any TRAPS patient. The other patients were her sons (aged 29 and 26 years) with similar findings in whom the authors also found a heterozygous C29R mutation.

Conclusion The authors report a novel C29R mutation in three patients of Turkish origin with TRAPS in whom the main clinical features were recurrent fever attacks, erythematous skin rash, conjunctivitis, myalgia and arthralgia. Treatment with steroids completely resolved the symptoms and lesions. 